\title{
The Effectiveness of Locomotion Interfaces Depends on Self-Motion Cues, Environmental Cues, and the Individual
}

\author{
Jonathan W. Kelly* Stephen B. Gilbert ${ }^{\dagger}$
}

lowa State University

\begin{abstract}
The proliferation of locomotion interfaces for virtual reality necessitates a framework for predicting and evaluating navigational success. Spatial updating - the process of mentally updating one's self-location during locomotion-is a core component of navigation, is easy to measure, and is sensitive to common elements of locomotion interfaces. This paper highlights three factors that influence spatial updating: body-based self-motion cues, environmental cues, and characteristics of the individual. The concordance framework, which characterizes locomotion interfaces based on agreement between body movement and movement through the environment, serves as a useful starting point for understanding the effectiveness of locomotion interfaces for enabling accurate navigation.
\end{abstract}

Index Terms: Human-centered computing-Human computer interaction (HCI) - Interaction paradigms_-Virtual reality

\section{INTRODUCTION}

Navigation is rarely the primary task in a virtual environment (VE). However, successful navigation supports primary tasks. For example, a prospective real estate buyer, whether touring a home virtually or in person, must be able to apprehend the spatial layout of the home in order to determine if it will fit their needs. Disorientation during the home tour will negatively affect the accuracy of the buyer's cognitive map of the home. We propose that navigational success in virtual reality (VR) depends on the self-motion cues afforded by the locomotion interface, environmental cues available in the VE, and characteristics of the individual navigator.

The research described herein focuses on spatial updating, which is a core cognitive component of navigation. Spatial updating is the process of keeping track of self-position and self-orientation during locomotion. Spatial updating failure is synonymous with disorientation. More advanced spatial cognitive processes, such as formation of a cognitive map, depend heavily on spatial updating. For example, developing a cognitive map of a house requires integration of separate spaces connected through spatial updating. At the extreme, if the user is disoriented, then the accuracy of the cognitive map will suffer severely. But even less extreme disruption of spatial updating will negatively impact cognitive map accuracy.

\section{ReLATED WORK}

This section highlights several factors that determine whether a locomotion interface will enable accurate spatial updating.

\subsection{Self-motion cues and the concordance framework}

There is broad evidence supporting the importance of walking in VR. For example, physically walking through a VE enhances presence [11] and reduces disorientation $[6,10]$ compared to other methods of locomotion that do not involve walking.

*e-mail: jonkelly@ iastate.edu

†e-mail:gilbert@iastate.edu
Walking generates a host of self-motion cues that facilitate spatial updating. These cues can be categorized as internal self-motion cues and external self-motion cues. Internal self-motion cues, herein referred to as body-based cues, are cues internal to the navigator, including vestibular stimulation caused by linear and angular accelerations, proprioceptive cues from movement of limbs and joints, and efferent copies of signals generated by motor cortex. External selfmotion cues are provided by optic flow and acoustic flow, defined as changes to the visual or acoustic array as a result of self-motion. Vection, or illusory self-motion, can occur When external self-moction cues are presented in the absence of internal self-motion cues.

Navigation research indicates that body-based cues play an outsize role in spatial updating. In one study [6], participants completed a triangle completion task in which they traveled to two waypoints before attempting to return to the path origin. Visual self-motion alone led to poor performance. Inclusion of body rotation at the waypoints led to performance equivalent to full walking. In another study [10], participants performing a virtual foraging task became disoriented without translational and rotational body-based cues. Although these studies disagree on the sufficiency of rotational cues, they agree on the importance of body movement for spatial updating.

Although real walking is possible in most modern VR systems, physical space limitations necessitate the use of a locomotion interface to explore all but the smallest VEs. Locomotion interfaces necessarily modify or eliminate self-motion cues in order to allow exploration of VEs beyond the tracked physical space. Given the importance of body-based self-motion cues to spatial updating, the concordance framework [2] (Figure 1) was developed to describe locomotion interfaces based on the extent to which body movement is concordant with movement through the VE.
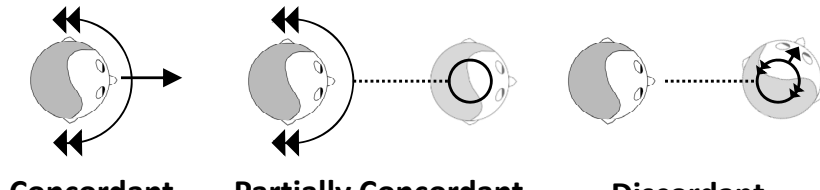

Concordant

Partially Concordant

Discordant

Figure 1: Examples illustrating the concordance framework for locomotion interfaces [2]. Walking (left) is concordant because body movement is consistent with movement through the VE. Teleporting to translate but using the body to rotate is partially concordant (middle). Teleporting to translate and rotate (right) is discordant, because the body is static during translations and rotations through the VE.

Concordant interfaces are those in which body movement is consistent with movement through the VE. Walking is the quintessential concordant interface. Partially concordant interfaces are those in which movement of the body is partially consistent with movement through the VE. An excellent example is the teleporting interface in which translation is achieved by teleporting but rotation is achieved by body rotation. Discordant interfaces are those in which body movement is uncoupled from movement through the VE. Joystick locomotion is a good example of a discordant interface because the user's body is stationary during locomotion through the VE. 
Research on variations of the teleporting interface supports the constructs defined by the concordance framework. Using the triangle completion task to measure spatial updating, performance is best when walking (concordant interface), worst when teleporting to translate and rotate (discordant interface), and in between when teleporting to translate and using the body to rotate (partially concordant) $[2,4]$. Furthermore, cognitive maps are more accurate after exploring through partially concordant teleporting compared to discordant teleporting [7].

The concordance framework is a starting point for understanding how locomotion interfaces affect navigation. A more complete understanding requires further research on how specific locomotion interfaces affect core navigation tasks such as spatial updating. For example, the finding that arm-swinging [8] increases spatial updating accuracy suggests that the concordance framework should be expanded to include arm movements. Careful research is needed to determine whether other interfaces that involve body movement, such as stepping in place [12] or even finger walking [5], convey benefits for spatial updating. Redirected walking [9] involves full body movement, but whether this leads to accurate spatial updating should determine its place in the concordance framework.

\subsection{Environmental cues}

Environmental cues can mitigate navigational deficiencies associated with partially concordant and discordant locomotion interfaces through a process known as piloting: navigation based on visible landmarks. For example, a recognizable university building provides an instantaneous position and orientation fix, which can correct for spatial updating errors that would otherwise accumulate over time.

In one study [2], triangle completion performance within a realistic classroom VE was superior to that within an open field VE, but the benefits of the classroom piloting cues were moderated by the locomotion interface, with the greatest benefit associated with a discordant teleporting interface, a smaller benefit associated with a partially concordant teleporting interface, and no benefit associated with walking. Surprisingly, benefits were only associated with spatial boundaries, such as walls or a fence, and not with landmarks scattered within the VE. Further research is needed to identify the specific types of environmental cues that are useful for mitigating disorientation caused by interface discordance.

\subsection{Individual differences}

The effectiveness of a locomotion interface also depends on characteristics of the individual. A recent study [1] explored individual differences using a triangle completion task with three interfaces: walking, partially concordant teleporting, and discordant teleporting. The task was conducted in an empty field VE and a classroom VE. Three groups of participants emerged. All groups performed well when walking. One group performed especially poorly when teleporting, and barely benefited from the classroom piloting cues. A second group also performed poorly when teleporting in the field, but improved considerably in the classroom. A third group did quite well overall and improved in the classroom compared to the field. Groups differed along several dimensions, including gender and video game experience, but only perspective-taking ability distinguished all the three groups. These results highlight the importance of predicting the effectiveness of locomotion interfaces for individual users. A future goal is to make interface recommendations to users based on their individual abilities and experiences.

\section{Conclusions}

Navigational success in VR depends on the self-motion cues afforded by the interface, environmental cues in the VE, characteristics of the individual navigator, and interactions between those variables. Research should expand by exploring interfaces that include body movements such as stepping, leaning, and arm movements, going beyond basic walking and body rotation. The concordance framework could provide a useful taxonomy to distinguish interfaces presented within the Locomotion Vault [3]. Furthermore, research on the interaction between locomotion interfaces and piloting cues should identify the critical features of useful environmental cues. For example, if boundaries are essential, what defines a boundary? Finally, longitudinal research should explore how user experience impacts navigation. Does interface training and experience lead to superior spatial updating? If so, do such benefits transfer to novel interfaces?

\section{ACKNOWLEDGMENTS}

Thanks to all those involved in this research for their help developing the ideas. This material is based upon work supported by the National Science Foundation under Grant No. CHS-1816029.

\section{REFERENCES}

[1] L. A. Cherep, J. W. Kelly, A. J. Miller, A. F. Lim, and S. B. Gilbert. Individual differences in teleporting through virtual environments. PsyArXiv, Dec 2020. doi: 10.31234/osf.io/b6cyd

[2] L. A. Cherep, A. F. Lim, J. W. Kelly, D. Acharya, A. Velasco, E. Bustamante, A. G. Ostrander, and S. B. Gilbert. Spatial cognitive implications of teleporting through virtual environments. Journal of Experimental Psychology: Applied, 26(3):480-492, 2020. doi: 10. 1037/xap0000263

[3] M. Di Luca, H. Seifi, S. Egan, and M. Gonzalez-Franco. Locomotion vault: the extra mile in analyzing vr locomotion techniques. In Proceedings of the 2021 CHI Conference on Human Factors in Computing Systems, CHI '21, 2021. doi: 10.1145/3411764.3445319

[4] J. W. Kelly, A. G. Ostrander, A. F. Lim, L. A. Cherep, and S. B. Gilbert. Teleporting through virtual environments: Effects of path scale and environment scale on spatial updating. IEEE Transactions on Visualization and Computer Graphics, 26(5):1841-1850, 2020. doi: 10.1109/TVCG.2020.2973051

[5] J.-S. Kim, D. Gračanin, K. Matković, and F. Quek. Finger walking in place (fwip): A traveling technique in virtual environments. In A. Butz, B. Fisher, A. Krüger, P. Olivier, and M. Christie, eds., Smart Graphics, pp. 58-69. Springer Berlin Heidelberg, Berlin, Heidelberg, 2008. doi: 10.1007/978-3-540-85412-8_6

[6] R. L. Klatzky, J. M. Loomis, A. C. Beall, S. S. Chance, and R. G. Golledge. Spatial updating of self-position and orientation during real, imagined, and virtual locomotion. Psychological Science, 9:293-298, 1998. doi: 10.1111/1467-9280.00058

[7] A. F. Lim, J. W. Kelly, N. C. Sepich, L. A. Cherep, G. C. Freed, and S. B. Gilbert. Rotational self-motion cues improve spatial learning when teleporting in virtual environments. In Proceedings of the Symposium on Spatial User Interaction, 2020. doi: 10.1145/3385959.3418443

[8] M. McCullough, H. Xu, J. Michelson, M. Jackoski, W. Pease, W. Cobb, W. Kalescky, J. Ladd, and B. Williams. Myo arm: Swinging to explore a ve. In Proceedings of the ACM SIGGRAPH Symposium on Applied Perception, SAP '15, p. 107-113. Association for Computing Machinery, New York, NY, USA, 2015. doi: 10.1145/2804408. 2804416

[9] N. C. Nilsson, T. Peck, G. Bruder, E. Hodgson, S. Serafin, M. Whitton, F. Steinicke, and E. S. Rosenberg. 15 years of research on redirected walking in immersive virtual environments. IEEE Computer Graphics and Applications, 38(2):44-56, 2018. doi: 10.1109/MCG.2018. 111125628

[10] R. A. Ruddle and S. Lessels. For efficient navigational search, humans require full physical movement, but not a rich visual scene. Psychological Science, 17(6):460-465, 1998. doi: 10.1111/j.1467-9280.2006. 01728.x

[11] M. Slater, M. Usoh, and A. Steed. Taking steps: The influence of a walking technique on presence in virtual reality. ACM Trans. Comput.-Hum. Interact., 2(3):201-219, Sept. 1995. doi: 10.1145/210079.210084

[12] J. N. Templeman, P. S. Denbrook, and L. E. Sibert. Virtual locomotion: Walking in place through virtual environments. Presence: Teleoperators and Virtual Environments, 8(6):598-617, 1999. doi: 10. $1162 / 105474699566512$ 